\title{
Beschäftigungsverbot bei Schwangeren? Darauf kommt es an!
}

\author{
Individuelles Beschäftigungsverbot oder Arbeitsunfähigkeit \\ in der Schwangerschaft? Die Grenze ist oft schwierig zu \\ ziehen, die Unterscheidung folgenschwer. Dem Arzt wird \\ allerdings ein gewisser Beurteilungsspielraum eingeräumt.
}

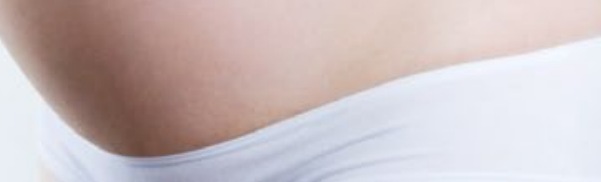

Ob ein individuelles Beschäftigungsverbot oder eine Arbeitsunfähigkeit (AU) ausgesprochen wird, ist schon aus finanzieller Hinsicht relevant, denn es hat unterschiedliche Konsequenzen für Arbeitgeber, Krankenkassen und Schwangere. Im Falle eines individuellen Beschäftigungsverbots zahlt der Arbeitgeber der Schwangeren das volle Entgelt.

\section{Kosten zu 100\% erstattet}

Die Kosten werden ihm zu 100\% von der Umlageversicherung U2 erstattet. Bei Arbeitsunfähigkeit zahlt der Arbeitgeber für sechs Wochen das volle Entgelt. Danach erhält die Schwangere Krankengeld von ihrer Krankenkasse, das allerdings nur etwa $70 \%$ des Nettogehalts beträgt. Arbeitgebern mit maximal 30 Beschäftigten werden bis zu 80\% der entstehenden Kosten aus der Umlageversicherung 1 erstattet. Ein individuelles Beschäftigungsverbot wird ausgesprochen, wenn das Leben oder die Gesundheit von Mutter oder Kind bei Fortdauer der Tätigkeit gefährdet ist. Ziel ist es, das Risiko für beide zu minimieren. Arbeitsunfähigkeit ergibt sich dagegen entweder aus einer Erkrankung oder einem Unfall ohne Kausalzusammenhang zur Schwangerschaft oder infolge eines pathologischen Schwangerschaftsverlaufs, etwa einer Präeklampsie, vorzeitiger Wehentätigkeit oder Blutungen. Laut Anneliese Bodemar von der Techniker Krankenkasse, Landesvertretung Rheinland-Pfalz, ist für die Entscheidung zwischen individuellem Beschäftigungsverbot und AU relevant, aus welchem Grund die Schwangere ihre Beschäftigung nicht ausüben sollte: wegen einer zusätzlichen Erkrankung, beispielsweise wegen einer Grippe, oder zu ihrem Schutz vor einer Überlastung durch die auszuübende Tätigkeit. Dennoch: Die Abgrenzung in der Praxis ist oft schwierig. In der Praxis wird dem
Arzt ein gewisser Beurteilungsspielraum eingeräumt. Dem ärztlichen Zeugnis über das Beschäftigungsverbot wird ein besonders hoher Stellenwert zugesprochen und das Urteil des Arztes ist zu respektieren, wenn die Entscheidung nachvollziehbar ist.

\section{Strafe bei nicht korrektem Verbot}

Stellt er allerdings ein individuelles Beschäftigungsverbot aus, das nicht korrekt ist, macht er sich strafbar, so Claudia Halstrick, Justiziarin beim Berufsverband der Frauenärzte, München. Ebenfalls wichtig: Besteht bereits ein individuelles Beschäftigungsverbot und es tritt zusätzlich Arbeitsunfähigkeit auf, muss das gemeldet werden, weil die Kosten aus unterschiedlichen Töpfen bezahlt werden

Fortbildungskongress FOKO 2013 der Frauenärztlichen BundesAkademie (FBA) 2013, Düsseldorf

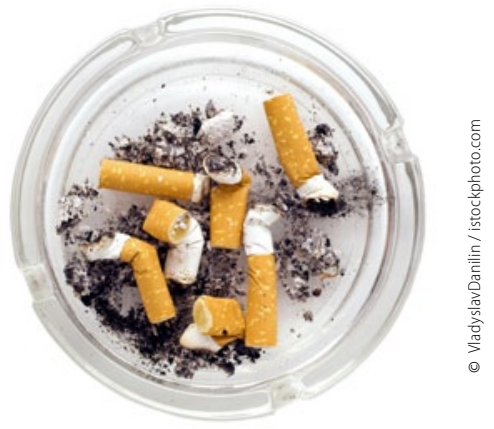

Das meldet der Deutsche Anwaltverein anlässlich eines aktuellen Urteils des Sozialgerichts Berlin. In der Sache ging es um eine Pflegehelferin, die von einer Raucherpause zu ihrer Arbeit zurückkehrte. Dabei stieß sie in der Eingangshalle des Seniorenheims mit dem Hausmeister zusammen. Diesem entglitt dabei ein Wassereimer, die Frau rutschte aus und brach sich den rechten Arm. Die
Pflegehelferin war der Ansicht, dass es sich um einen Arbeitsunfall handelte. Die Berufsgenossenschaft lehnte die Anerkennung eines Arbeitsunfalls ab. Hiergegen klagte die Frau.

Ohne Erfolg: Rauchen sei persönliche Angelegenheit ohne sachlichen Bezug zur Berufstätigkeit, so die Richter. Deshalb bestehe bei einer Verletzung auch kein Anspruch auf Heilbehandlung, Verletztengeld oder Rente gegenüber der gesetzlichen Unfallversicherung. Es sei die private Entscheidung jedes Einzelnen, ob er zum Rauchen gehe oder nicht. Das Rauchen sei insbesondere nicht mit der Nah- rungsaufnahme vergleichbar. Essen und Trinken seien unter anderem nötig, um die Arbeitskraft aufrechtzuerhalten. Deshalb sei zwar der Weg zur Kantine versichert, nicht aber der Weg von und zur Raucherpause.

(cW)

Az.: S 68 U 577/12; Ärzte Zeitung, 26.2.2013 